Pacific Journal of Mathematics

ON THE DEGENERACY OF THE KEPLER PROBLEM 


\title{
ON THE DEGENERACY OF THE KEPLER PROBLEM
}

\author{
Victor A. DUlock aNd Harold V. McIntosh
}

In an earlier paper we have shown a method which may be used to construct an $S U(n)$ group as the symmetry group of the harmonic oscillator in classical mechanics. The method is applicable to quadratic Hamiltonians, and was applied in a subsequent paper to the charged harmonic oscillator in a magnetic field. We now apply the technique to the Kepler problem, which may be made equivalent to a harmonic oscillator by a suitable transformation. An $S U(3)$ group is found, generated by constants of the motion which are the angular momentum, the Runge vector, which points to the perihelion, and a vector along the line of nodes. Different groups are found by separation in spherical and parabolic coordinates, while yet another group is found by inspection in parabolic coordinates. One purpose of our investigation is to find symmetry groups for quantum mechanical problems. While our results dispel the thought that there might not be sets of constants of the motion closed with respect to Poisson Brackets and thus generating a Lie symmetry group, they do show that the functional relationship involved may make it very difficult to use the correspondence principle to construct satisfactory quantum mechanical operators. Our $S U(3)$ symmetry group is not isomorphic to the $R(4)$ symmetry group of the hydrogen atom found by Fock and Bargmann; the angular momentum and Runge vector are nonlinear functions of a subset of the generators of our $S U(3)$ group. It is not possible to find an operator generalization of these functions which can be satisfied by irreducible representations of $S U(3)$ and $R(4)$.

Among the textbook problems of quantum mechanics, the harmonic oscillator and the Kepler problem have been particularly interesting because they both display a considerably higher degree of degeneracy than would be expected on the basis of their spherical symmetry. Even the two dimensional versions of these two problems display much more than the two-fold degeneracy which would be expected on the basis of the circular symmetry of their potentials.

The origins of this excessive, or "accidental" degeneracy have been known for some time, since Fock [9] in 1935 showed the equivalence of the bound state motion of the Kepler problem to force free motion on the surface of a hypersphere. The four-dimensional rotation group thus placed in evidence by a stereographic projection from momentum space has irreducible representations which adequately account for the observed degeneracy. Demkov [6] has recently inquired into the 
philosophical aspects of the question, for it still remains an open question as to when one has discovered "the" symmetry group of a Hamiltonian.

Laporte and Rainich [16] showed how stereographic parameters could lead to accidental degeneracy in much the same way as Fock's analysis, since many types of motion could be shown to be equivalent to force free motion on a hypersphere, and thus would possess the hidden symmetry of a higher dimensional rotation group. By using such methods Jauch and Hill [15], Hill [13], and Saenz [23] subsequently discussed the harmonic oscillator, Kepler problem, and the rigid rotator.

Although one is trying to solve an essentially quantum mechanical problem, one is eventually drawn to its classical analog, for it seems that the degeneracy in the quantum mechanical version is closely related to the existence of bounded closed orbits in the classical problem. In this respect it seems that the three problems mentioned are substantially the only ones possessing accidental degeneracy [23, Introduction], for their potentials are the only ones possessing spherical symmetry for which the orbits are bounded and closed. There are sufficient conditions in the statement of this latter theorem to provide a few loopholes ... cyclotron motion in a uniform magnetic field being an example which we have discussed recently [7].

In order to understand the circumstances under which degeneracy might arise, and its relation to the bounded, closed orbits we have sought a different principle from the hidden symmetry introduced by stereographic parameters, which would yield a classical symmetry group. It has been introduced in an earlier paper [8], in which we dealt with a quadratic Hamiltonian. By the use of the Poisson Bracket (P. B.), such Hamiltonians may be regarded as linear operators acting on functions of the phase space coordinates and momenta. Their eigenvalues occur in negative pairs, which allows the construction of constants of the motion as products of the eigenfunctions belonging to a negative pair of eigenvalues. In this way the possible constants of the motion may be accurately determined, and the Lie groups which they determine may be investigated. Once this is done quantum mechanical generalizations may be considered.

The result of this approach is that there will be a symmetry group isomorphic to an unitary unimodular group $S U(n)$, whenever there are degenerate eigenvalues in the operator representation of the Hamiltonian. Such degenerate eigenvalues may occur on more general grounds than the spherical symmetry of the potential. In fact, for the isotropic harmonic oscillator they occur on the simple grounds that an $n$-dimensional oscillator is separable into $n$ identical 1-dimensional oscillators. 
Thus a permutation symmetry of the Hamiltonian is sufficient to invoke a unitary unimodular symmetry of the entire system.

Since this method is based on the properties of linear operators, it would seem at first sight that it would be applicable only to quadratic Hamiltonians. However, one has to think of the Hamilton-Jacobi theory, by which all mechanical problems are reduced in principle to force free motion in some nonEuclidean space. A method similar to this has been used by Gardner [11] in his theory of adiabatic invariants. The problems remaining, although quite nontrivial, are to identify the topological properties of this final space and to effectively discover the change of coordinates effecting the transformation.

If we grant the reduction of mechanical problems to force free motion, then by introducing polar coordinates we can obtain circular orbits in yet another nonEuclidean space, and effectively a quadratic Hamiltonian. Thus it would seem that potentially there is an $S U(n)$ symmetry group for every problem in classical mechanics. Outrageous as such an assertion seems at first sight, it happens that the problem lies not with the existence, but rather with the utility of such a symmetry group. By this we mean that the transformation which yields the eventual equivalent harmonic oscillator may be very complicated, and in particular will not be a single valued function. Thus, even though a classical symmetry group may exist, it may be quite impossible to use the correspondence principle to construct an analogous group of operators for the quantum mechanical version of the problem. This failing is already apparent for the anisotropic harmonic oscillator when the frequencies are incommensurable.

By applying these methods to the Kepler problem, with its known degeneracies, this approach is further illuminated. Although Fock's paper represented the first identification of a dynamical symmetry group for the Kepler problem, constants of the motion themselves had been known at least from the time that Newton formulated the problem by means of the calculus, and were a conspicuous feature of most textbooks on analytical mechanics [24]. In addition to the angular momentum

$$
\vec{L}=\vec{r} \times \vec{p},
$$

which stands perpendicular to the plane of the orbit, the Runge vector

$$
\vec{R}=\frac{\vec{L} \times \vec{p}}{m Z e^{2}}-\frac{\vec{r}}{r}
$$

was known to be a constant of the motion. It is a vector pointing to the perihelion and whose magnitude is the eccentricity of the orbit. The fact that it is constant stabilizes the orbit. When the $1 / r$ attractive 
potential is perturbed, the perihelion processes, and the Runge vector is no longer constant. Only by destroying the spherical symmetry of the potential is the plane of the orbit altered, and the angular momentum rendered nonconstant.

Bargmann [2], in a commentary on Fock's paper, showed that the components of these two vectors generated the Lie group $R(4)$, which was known to be the symmetry group of the Kepler problem on account of Fock's stereographic projection from the surface of a hypersphere. However, Pauli [21] had used the commutation rules for the components of these vectors to solve the quantum mechanical problem of the hydrogen atom already in 1926, extending an earlier result of Lenz [17], and 0 . Klein [14] had noted that the commutation rules were those of the orthogonal group $R(4)$.

Most of these authors had noted that between the two vectors $L$ and $R$ there were six constants of the motion, in addition to the Hamiltonian $H$, itself. The Hamilton-Jacobi theory only requires five, in addition to $H$. The existence of two identities

$$
\vec{L} \cdot \vec{A}=0
$$

and

$$
L^{2}+A^{2}=H^{2}
$$

where

$$
\vec{A}=\vec{R} / \sqrt{-2 E}
$$

not only reconciles the number of constants, but is useful in using the matrix mechanics to solve the hydrogen atom. The fact that there are already two redundant constants involved in defining a symmetry group obscures the fact that the Kepler problem possesses other significant constants, such as a vector pointing along the line of nodes.

The scheme which we have proposed would exhibit $S U(3)$ as a symmetry group of the Kepler problem, with eight constants of the motion, in addition to $H$. This is in fact the result of our analysis, the additional redundant constants fixing the line of nodes. Since there is no isomorphism between $S U(3)$ and $R(4)$, and in addition the dimensions of their irreducible representations are different, we are rather embarassed by our new found symmetry group. As it happens, there is a functional relation between some of the generators of $S U(3)$ and those of $R(4)$, such that the commutation rules of both groups will be satisfied. The functional relationship, which works with respect to Poisson Brackets, will apparently not work for noncommutative operators and commutator brackets. Although we have avoided a 
contradiction in this way, there is evidence of a deeper relation, which needs further careful study. For instance, one is reminded of the relation between Coulomb integrals and Wigner coefficients when he sees that one could replace the angular momentum by the radial action in the group $S U(3)$ [20].

In addition to the Kepler problem in three dimensions, many of the authors we have cited have studied the two-dimensional version obtained by taking the orbital plane. Alliluev [1] considered the problem generally in $n$-dimensions, while Loudon [18] has obtained an unexpected degeneracy even in one dimension. Also, several authors have treated variants of the Kepler problem, such as introducing rotating coordinates to overcome relativistic corrections $[3,4]$ or considering a superimposed uniform force field [22].

The choice of a coordinate system in which to separate the Kepler problem influences the nature of the symmetry group which we obtain. Since the essential features of the problem are already present in two dimensions, we have separated the two-dimensional Hamiltonian in both polar and parabolic coordinates. The generators which we obtain for an $S U(2)$ group in each case are quite different, although functionally related. In fact, we obtain the Runge vector directly in the parabolic coordinates only by making a fortuitous substitution which occurs to us, and not by following our theory rigorously.

2. The two dimensional case in polar coordinates. The Hamiltonian for the two dimensional Kepler problem in polar coordinates is

$$
H=(1 / 2)\left(P_{r}^{2}+P_{\phi}^{2} / r^{2}\right)-1 / r
$$

where the mass and the force constant have been chosen equal to unity. The corresponding Hamilton-Jacobi equation is

$$
\left(\frac{\partial S_{r}}{\partial r}\right)^{2}+\frac{1}{r^{2}}\left(\frac{\partial S_{\phi}}{\partial \phi}\right)^{2}-2(W+1 / r)=0
$$

where $W$ is the total energy and the characteristic function $S$ is given by

$$
S(r, \phi)=S_{r}(r)+S_{\phi}(\phi)
$$

Hence

$$
P_{r}=\partial S / \partial r
$$

and

$$
P_{\phi}=\partial S / \partial \phi
$$


Since the partial differential equation (6) has no $\phi$ dependence $P_{\phi}=$ $\partial S / \partial \phi$ is a constant and is, in fact, the angular momentum of the system. Solving (6) for $P_{r}=\partial S / \partial r$ one finds

$$
\partial S / \partial r=P_{r}=\left[2 W+(2 / r)-P_{\phi}^{2} / r^{2}\right]^{1 / 2} .
$$

The action variables $J_{r}$ and $J_{\phi}$ are given by the integrals

$$
\begin{aligned}
J_{r} & =\int P_{r} d r \\
J_{\phi} & =\int P_{\phi} d \phi .
\end{aligned}
$$

The last equation can be integrated immediately since the integral over one cycle is simply an integral from 0 to $2 \pi$, hence

$$
J_{\phi}=2 \pi P_{\phi} \text {. }
$$

The integral in (10a) can be evaluated by a contour integration as described in Born [5] and the result is

$$
J_{r}=\left[2 /(-2 W)^{1 / 2}\right]-J_{\phi} .
$$

The angle variables $w_{r}$ and $w_{\phi}$ which are canonical coordinates and conjugate to $J_{r}$ and $J_{\phi}$ are given by

$$
w_{r}=\partial S / \partial J_{r}=\int\left(\partial P_{r} / \partial J_{r}\right) d r
$$

and

$$
w_{\phi}=\partial S / \partial J_{\phi}=\int\left(\partial P_{r} / \partial J_{\phi}\right) d r+\int\left(\partial P_{\phi} / \partial J_{\phi}\right) d \phi
$$

Upon performing the integrations

$$
\begin{gathered}
w_{r}=(u-\varepsilon \sin u) / 2 \pi \\
w_{\phi}=\left[u-\varepsilon \sin u-\tan ^{-1}(y / x)+\phi\right] / 2 \pi,
\end{gathered}
$$

where the elliptic anomaly $u$ is defined through

$$
r=a(1-\varepsilon \cos u),
$$

$a$ being the semi-major axis and $\varepsilon$ being the eccentricity of the ellipse,

$$
\begin{gathered}
a=-W / 2 \\
\varepsilon^{2}=1+2 W P_{\phi}^{2} .
\end{gathered}
$$

By choosing $\phi_{0}$ to be the azimuth of the perihelion, 
(18a)

(18b)

$$
\begin{aligned}
& x=r \cos \left(\phi-\phi_{0}\right) \\
& y=r \sin \left(\phi-\phi_{0}\right),
\end{aligned}
$$

and $(14 b)$ reduces to

$$
w_{\phi}=\left(u-\varepsilon \sin u+\phi_{0}\right) / 2 \pi .
$$

Solving (12) for the energy in terms of $J_{r}$ and $J_{\phi}$

$$
W=2 \pi^{2} /\left(J_{r}+J_{\phi}\right)^{2}
$$

and hence the frequencies $\nu_{r}$ and $\nu_{\phi}$ are degenerate i.e.,

$$
\nu_{r}=\nu_{\phi}=\partial W / \partial J_{r}=\partial W / \partial J_{\phi} \text {. }
$$

Thus the quantities $a a^{*}, b b^{*}, a b^{*}$, and $a^{*} b$ are constants of the motion where

$$
a=\sqrt{J_{r} / 2 \pi} \exp \left(2 \pi i w_{r}\right)
$$

and

$$
b=\sqrt{J_{\phi} / 2 \pi} \exp \left(2 \pi i w_{\phi}\right) .
$$

These quantities satisfy the following P.B. relations

$$
\left\{a, a^{*}\right\}=\left\{b, b^{*}\right\}=i
$$

here one uses the fact the $J^{\prime} s$ and $w^{\prime} s$ form a set of canonical coordinates and momenta.

Using the technique of our earlier paper [8], we take the following four quantities as constants of the motion:

$$
\begin{aligned}
& H=a a^{*}+b b^{*} \\
& D=a a^{*}-b b^{*} \\
& K=a b^{*}+a^{*} b \\
& L=i\left(a b^{*}-a^{*} b\right) .
\end{aligned}
$$

Of these constants $H$ commutes with the other three and is a function of the Hamiltonian, namely, it is inversely proportional to the square root of the energy. The three constants $K, D$, and $L$ satisfy the commutation relations of the group $S U(2)$ or $R(3)$,

$$
\begin{aligned}
& \{K, D\}=2 L \\
& \{L, K\}=2 D \\
& \{D, L\}=2 K .
\end{aligned}
$$


Although they have no simple interpretation as constants of the motion, one can make a mapping of these constants which preserves the commutation rules. The new constants can be interpreted as the angular momentum and the two components of the Runge vector [23]. The mapping is

$$
\begin{aligned}
K \rightarrow K^{\prime} & =K[2+(H+D) /(H-D)]^{1 / 2} \\
L \rightarrow L^{\prime} & =L[2+(H+D) /(H-D)]^{1 / 2} \\
D \rightarrow D^{\prime} & =H-D .
\end{aligned}
$$

These constants satisfy the rules

$$
\begin{aligned}
& \left\{K^{\prime}, D^{\prime}\right\}=-2 L^{\prime} \\
& \left\{D^{\prime}, L^{\prime}\right\}=-2 K^{\prime} \\
& \left\{L^{\prime}, K^{\prime}\right\}=-2 D^{\prime} .
\end{aligned}
$$

They are a direct consequence of the commutation rules for $K, L$, and $D$ so that this mapping is valid for any $S U(2)$ group where use must be made of the identity

$$
H^{2}=K^{2}+L^{2}+D^{2} .
$$

$K^{\prime}$ is essentially the $x$ component of the vector $\vec{P}$ where

$$
\vec{P}=\vec{R} / \sqrt{-2 E}=(-\varepsilon / \sqrt{-2 E})\left|\begin{array}{c}
\cos \phi_{0} \\
\sin \phi_{0}
\end{array}\right|
$$

and $\vec{R}$ is the Runge vector, while $L^{\prime}$ is the $y$ component and $D^{\prime}$ is the angular momentum.

3. The two dimensional Kepler problem in parabolic coordinates. In parabolic coordinates, defined by

$$
\begin{aligned}
& x=\mu \nu \\
& y=\left(\mu^{2}-\nu^{2}\right) / 2
\end{aligned}
$$

and where $m=e=1$, the Kepler Hamiltonian is

$$
H=\left[P_{\mu}^{2}+P_{\nu}^{2}-4\right] /\left[2\left(\mu^{2}+\nu^{2}\right)\right] .
$$

From the Hamilton-Jacobi equation

$$
\begin{aligned}
& P_{\mu}=\left[2 W \mu^{2}+2 \alpha_{1}\right]^{1 / 2} \\
& P_{\nu}=\left[2 W \nu^{2}+2 \alpha_{2}\right]^{1 / 2}
\end{aligned}
$$


where

$$
\alpha_{1}+\alpha_{2}=2 \text {. }
$$

The action variables defined by

$$
J_{i}=\int_{0} P_{i} d q_{i}
$$

give

$$
J_{\mu}=-2 \pi \alpha_{1} / \sqrt{-2 W}
$$

and

$$
J_{\nu}=-2 \pi \alpha_{2} / \sqrt{-2 W}
$$

Solving Eqs. (32) and (35) for $\alpha_{1}$ and $\alpha_{2}$ and equating the solutions

$$
-J_{\mu} \sqrt{-2 W} /(2 \pi)=\left[P_{\mu}^{2}-2 W \mu^{2}\right] / 2
$$

and

$$
-J_{\nu} \sqrt{-2 W} /(2 \pi)=\left[P_{\nu}^{2}-2 W \nu^{2}\right] / 2
$$

Hence

$$
J_{\mu}=-\pi \sqrt{-2 W}\left[\mu^{2}+P_{\mu}^{2} /(-2 W)\right]
$$

and

$$
J_{\nu}=-\pi \sqrt{-2 W}\left[\nu^{2}+P_{\nu}^{2} /(-2 W)\right] \text {. }
$$

The form of these latter two equations suggests that two new variables be defined

$$
\begin{aligned}
& a=\mu+i P_{\mu} / \sqrt{-2 W} \\
& b=\nu+i P_{\nu} / \sqrt{-2 W},
\end{aligned}
$$

so that

$$
\begin{aligned}
& J_{\mu}=-\pi \sqrt{-2 W} a a^{*} \\
& J_{\nu}=-\pi \sqrt{-2 W} b b^{*} .
\end{aligned}
$$

Both $a$ and $b$ are very similar to the harmonic oscillator operators introduced in our earlier paper [8] and by analogy one would expect to find that quantities proportional to $a b^{*}$ and $a^{*} b$ are also constants of the motion. This is in fact the case. Taking real and imaginary parts of these quantities and normalizing, the constants are

$$
K=-\left[a b^{*}+a^{*} b\right] \sqrt{-2 W} / 2
$$


and

$$
L=-i\left[a b^{*}-a^{*} b\right] \sqrt{-2 W} / 2 .
$$

The third independent constant can be chosen to be the difference between $J_{\mu}$ and $J_{\nu}$

$$
D=\left[a a^{*}-b b^{*}\right] \sqrt{-2 W} / 2 .
$$

It is not difficult to verify that these constants satisfy the commutation relations of the generators of $S U(2)$ or $R(3)$ i.e.,

$$
\begin{gathered}
\{K, L\}=-2 D \\
\{L, D\}=-2 K \\
\{D, K\}=-2 L,
\end{gathered}
$$

where

$$
\left\{a^{*}, a\right\}=\left\{b^{*}, b\right\}=2 i / \sqrt{-2 W} .
$$

It should be noted that in this case the angle variables $w_{\mu}$ and $w_{2}$ were not used. This occurred only because a similarity was noted between the constants of the motion $J_{\mu}$ and $J_{\nu}$ and the constants of the motion for the harmonic oseillator. Implicit in the definitions of $a$ and $b$ are two variables, denote $\beta$ and $\gamma$, which are conjugate to $J_{\mu}$ and $J_{\nu}$. These variables are

$$
\beta=\frac{\tan ^{-1}\left(P_{\mu} / \sqrt{-2 W \mu}\right)}{2 \pi}
$$

and

$$
\gamma=\frac{\tan ^{-1}\left(P_{\nu} / \sqrt{-2 W \nu}\right)}{2 \pi}
$$

It is easy to verify that these are conjugate to the action variables for

$$
\begin{aligned}
& \left\{\gamma, J_{\nu}\right\}=\left\{\beta, J_{\mu}\right\}=1 \\
& \left\{\gamma, J_{\mu}\right\}=\left\{\beta, J_{\nu}\right\}=0 .
\end{aligned}
$$

However if one evaluates the formulas for the angle variables one does not obtain the same results. It should not be surprising to find a set of coordinates conjugate to the action variables which are not the angle variables because for a given set of momenta the set of canonical coordinates is not unique. This occurs because one can always add a total time derivative to the Lagrangian, which is a function 
of $q, \dot{q}$, and $t$.

The constants $K, L$, and $D$ have remarkably simple interpretations. $K$ and $D$ are the $x$ and $y$ components of the vector $\vec{P}=\vec{R} \sqrt{-2 W}$ where $\vec{R}$ is the Runge vector, while $\vec{L}$ is the angular momentum. This can be verified by substituting equation (38) into equations (40) and then transforming either to rectangular or polar coordinates.

4. The Kepler problem in three dimensions. Using the same units as in the previous case, the Hamiltonian for the Kepler problem in three dimensions is

$$
H=\left[P_{r}^{2}+P_{\theta}^{2} / r^{2}+P_{\phi}^{2} /\left(r^{2} \sin ^{2} \theta\right)\right] / 2-1 / r .
$$

Setting up the Hamilton-Jacobi equation and separating gives

$$
\begin{aligned}
& P_{\phi}=\partial S / \partial \phi=\alpha_{\phi}=\text { constant } \\
& P_{\theta}=\partial S / \partial \theta=\left[\alpha_{\theta}^{2}-\alpha_{\phi}^{2} / \sin ^{2} \theta\right]^{1 / 2}
\end{aligned}
$$

and

$$
P_{r}=\partial S / \partial r=\left[2 W+2 / r-\alpha_{\theta}^{2} / r^{2}\right]^{1 / 2},
$$

where $\alpha_{\theta}$ and $\alpha_{\phi}$ are separation constants, $\alpha_{\phi}$ being the magnitude of the $z$ component of the angular momentum and $\alpha_{\theta}$ the magnitude of total angular momentum. Since the potential is central the orbit is planar and the normal to the plane is necessarily parallel to the angular momentum. Denoting by $\beta$ the dihedral angle which the orbital plane makes with the $x y$ plane one can deduce that

$$
\cos \beta=\alpha_{\phi} / \alpha_{\theta} \text {. }
$$

Solving for the action variables gives

$$
\begin{aligned}
& J_{\phi}=2 \pi \alpha_{\phi}=2 \pi P_{\phi} \\
& J_{\theta}=2\left[\alpha_{\theta}-\alpha_{\phi}\right] \\
& J_{r}=-J_{\theta}-J_{\phi}+2 / \sqrt{-2 W}
\end{aligned}
$$

and from the last equation it follows that

$$
W=-2 \pi^{2} /\left[J_{r}+J_{\theta}+J_{\phi}\right]^{2} .
$$

In the plane of the orbit we introduce the rectangular coordinates $\mu$ and $\nu$, where the $\mu$ axis is along the major axis of the ellipse and the origin coincides with the center of force and the origin of the polar system. In this system

$$
\mu=r \cos \left(\psi-\psi_{0}\right)
$$


(50b)

$$
\nu=r \sin \left(\psi-\psi_{0}\right),
$$

where $\psi$ is an angle measured from the $\mu$ axis to the particle and $\psi_{0}$ is the angle to the perihelion.

The angle variables are

$$
\begin{gathered}
w_{r}=\int\left(\partial P_{r} / \partial J_{r}\right) d r=[u-\varepsilon \sin u] / 2 \pi \\
w_{\theta}=\int\left(\partial P_{r} / \partial J_{\theta}\right) d r+\int\left(\partial P_{\theta} / \partial J_{\theta}\right) d \theta \\
=\left[u-\varepsilon \sin u-\tan ^{-1}(\nu / \mu)+\sin ^{-1}(\cos \theta / \sin \beta)\right] / 2 \pi \\
w_{\phi}=\int\left(\partial P_{r} / \partial J_{\phi}\right) d r+\int\left(\partial P_{\theta} / \partial J_{\phi}\right) d \theta+\int\left(\partial P_{\phi} / \partial J_{\phi}\right) d \phi \\
=\left[u-\varepsilon \sin u-\tan ^{-1}(\nu / \mu)+\sin ^{-1}(\cos \theta / \sin \beta)\right. \\
\left.-\sin ^{-1}(\cot \theta / \tan \beta)+\phi\right] / 2 \pi
\end{gathered}
$$

where $u$ is defined in (15) and $\varepsilon$ in (17). Using equations (50) it follows that

$$
\tan ^{-1}(\nu / \mu)=\psi-\psi_{0} .
$$

It follows from Fig. 1 that $\sin ^{-1}(\cot \theta / \tan \beta)$ is the projection of the

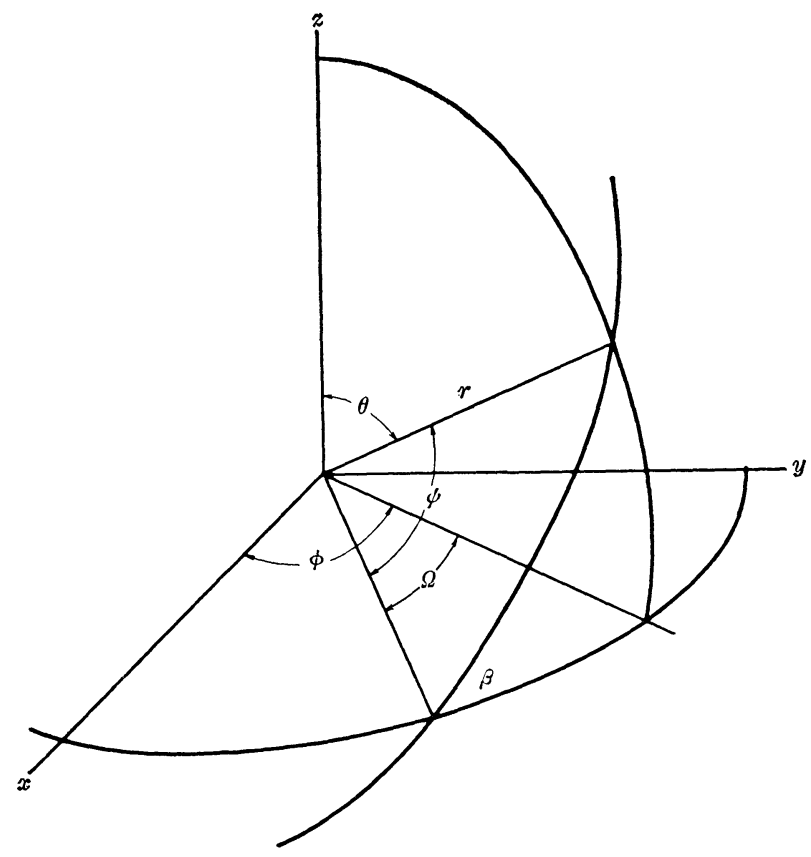

FIGURE 1.

Three dimensional coordinate system for the Kepler problem. 
angle $\psi$ onto the plane in which $\phi$ is measured i.e., the $x y$ plane, which shall be denoted $\Omega$. It also follows that $\sin ^{-1}(\cos \theta / \sin \beta)$ is the angle $\psi$, where the line of nodes has been chosen to coincide with the major axis of the ellipse. Hence, the line of nodes makes an angle $(\phi-\Omega)$ with the $x$ axis. Rewriting (51b) and (51c) results in the following set of equations,

$$
\begin{aligned}
& w_{\theta}=\left[u-\varepsilon \sin u+\psi_{0}\right] / 2 \pi \\
& w_{\phi}=\left[u-\varepsilon \sin u+\psi_{0}+\phi-\Omega\right] / 2 \pi .
\end{aligned}
$$

By defining

$$
\begin{aligned}
& a=\sqrt{J_{r} / 2 \pi} \exp \left(2 \pi i w_{r}\right) \\
& b=\sqrt{J_{\theta} / 2 \pi} \exp \left(2 \pi i w_{\theta}\right) \\
& c=\sqrt{J_{\phi} / 2 \pi} \exp \left(2 \pi i w_{\phi}\right)
\end{aligned}
$$

one finds the following nine constants of the motion: $a a^{*}, b b^{*}, c c^{*}, a b^{*}$, $a^{*} b, b c^{*}, b^{*} c, a c^{*}$, and $a^{*} c$. These constants are the generators of the group $S U(3)$. The constant angles $\beta, \psi_{0}$, and $(\phi-\Omega)$ and also the sum $\left(\phi-\Omega+\psi_{0}\right)$ can be expressed in terms of the constants,

$$
\begin{aligned}
\sin \psi_{0} & =i\left[a b^{*}-a^{*} b\right] / 2 \sqrt{a a^{*} b b^{*}} \\
\cos \psi_{0} & =\left[a b^{*}+a^{*} b\right] / 2 \sqrt{a a^{*} b b^{*}} \\
\sin (\phi-\Omega) & =i\left[b c^{*}-b^{*} c\right] / 2 \sqrt{b b^{*} c c^{*}} \\
\cos (\phi-\Omega) & =\left[b c^{*}+b^{*} c\right] / 2 \sqrt{b b^{*} c c^{*}} \\
\sin \left(\phi-\Omega-\psi_{0}\right) & =i\left[a c^{*}-a^{*} c\right] / 2 \sqrt{a a^{*} c c^{*}} \\
\cos \left(\phi-\Omega+\psi_{0}\right) & =\left[a c^{*}+a^{*} c\right] / 2 \sqrt{a a^{*} c c^{*}}
\end{aligned}
$$

and

$$
\cos \beta=c c^{*} /\left(b b^{*}+c c^{*}\right) .
$$

Generally, the rotation group in four dimensions, $R(4)$ is taken to be the symmetry group of the Kepler problem. Its generators are the three components of the angular momentum vector $\vec{L}$ and the three components of the normalized Runge vector $\vec{P}$, which is defined in terms of $\vec{R}$ in (29). The rectangular components of $\vec{P}$ are

$$
\vec{P}=\frac{\varepsilon}{\sqrt{-2 W}}\left|\begin{array}{c}
\cos \psi_{0} \cos (\phi-\Omega)-\cos \beta \sin \psi_{0} \sin (\phi-\Omega) \\
-\cos \psi_{0} \sin (\phi-\Omega)-\cos \beta \sin \psi_{0} \cos (\phi-\Omega) \\
\sin \beta \sin \psi_{0}
\end{array}\right| .
$$


For $\vec{L}$ one has

$$
\vec{L}=\frac{J_{\theta}+J_{\phi}}{2 \pi}\left|\begin{array}{l}
\sin \beta \sin (\phi-\Omega) \\
\sin \beta \cos (\phi-\Omega) \\
\cos \beta
\end{array}\right|
$$

Using equations (55) one can express the generators of $R(4)$ in terms of the generators of $S U(3)$ as follows:

$$
\begin{aligned}
P_{x}= & \sqrt{a a^{*}+2 b b^{*}+2 c c^{*}}\left[\left(a b^{*}+a^{*} b\right)\left(b c^{*}+b^{*} c\right)\left(b b^{*}+c c^{*}\right)\right. \\
& \left.+\left(a b^{*}-a^{*} b\right)\left(b c^{*}-b^{*} c\right) c c^{*}\right] /\left[4 b b^{*} \sqrt{c c^{*}}\left(b b^{*}+c c^{*}\right)\right] \\
P_{y}= & -i \sqrt{a a^{*}+2 b b^{*}+2 c c^{*}}\left[\left(a b^{*}+a^{*} b\right)\left(b c^{*}-b^{*} c\right)\left(b b^{*}+c c^{*}\right)\right. \\
& \left.+\left(a b^{*}-a^{*} b\right)\left(b c^{*}+b^{*} c\right) c c^{*}\right] /\left[4 b b^{*} \sqrt{c c^{*}}\left(b b^{*}+c c^{*}\right)\right]
\end{aligned}
$$

(58c) $P_{z}=i \sqrt{\left(a a^{*}+2 b b^{*}+2 c c^{*}\right)\left(b b^{*}+2 c c^{*}\right)}\left(a b^{*}+a^{*} b\right) / 2\left(b b^{*}+c c^{*}\right)$

(58d) $L_{x}=i\left(b c^{*}-b^{*} c\right) \sqrt{b b^{*}+2 c c^{*}} / 2 \sqrt{c c^{*}}$

(58e) $L_{y}=\left(b c^{*}+b^{*} c\right) \sqrt{b b^{*}+2 c c^{*}} / 2 \sqrt{c c^{*}}$

and

(58f) $L_{z}=c c^{*}$.

These six quantities satisfy the relations

$$
\begin{aligned}
& \left\{L_{i}, P_{j}\right\}=-\varepsilon_{i j k} P_{k} \\
& \left\{L_{i}, L_{j}\right\}=-\varepsilon_{i j k} L_{k} \\
& \left\{P_{i}, P_{j}\right\}=-\varepsilon_{i j k} L_{k}
\end{aligned}
$$

which define $R(4)$, once again directly from the commutation rules for $S U(3)$.

The process can be inverted and the generators of $S U(3)$ can be expressed in terms of the components of $\vec{P}$ and $\vec{L}$. These are

(60a) $\quad a a^{*}=1 / \sqrt{-2 W}-\sqrt{L_{x}^{2}+L_{y}^{2}}-L_{z}$

(60b) $\quad b b^{*}=\sqrt{L_{x}^{2}+L_{y}^{2}}$

(60c) $\quad c c^{*}=L_{z}$

(60d) $\quad a b^{*}=\sqrt{L_{x}^{2}+L_{y}^{2}}\left[1 / \sqrt{-2 W}-\sqrt{L_{x}^{2}+L_{y}^{2}}-L_{z}\right]^{1 / 2} \exp \left(-i \psi_{0}\right)$

(60e) $\quad b c^{*}=\left[L_{z} \sqrt{L_{x}^{2}+L_{y}^{2}}\right]^{1 / 2} \exp (-i(\phi-\Omega))$

(60f) $\quad a c^{*}=\sqrt{L_{z}}\left[1 / \sqrt{-2 W}-\sqrt{L_{x}^{2}+L_{y}^{2}}-L_{z}\right]^{1 / 2} \exp \left(-i\left(\phi-\Omega+\psi_{0}\right)\right)$ where 
(61a) $\phi-\Omega=\tan ^{-1}\left(L_{x} / L_{y}\right)$

$$
\psi_{0}=\tan ^{-1}\left\{P_{z} L_{y} \sqrt{L_{x}^{2}+L_{y}^{2}+L_{z}^{2}} /\left[P_{x}\left(L_{x}^{2}+L_{y}^{2}\right)+P_{z} L_{x} L_{z}\right]\right\}
$$

and

$$
\beta=\tan ^{-1}\left(\sqrt{L_{x}^{2}+L_{y}^{2}} / L_{x}\right)
$$

5. Conclusion. It has been known for a rather long time that the Kepler problem is somehow equivalent to the harmonic oscillator. Quantum mechanically the relation has been exploited by Schwinger in his course lectures as a means of solving the problem of the hydrogen atom for several years. One technique is to note the resemblance between the Laguerre polynomials and the Hermite polynomials [19], and by making the substitution $r=\rho^{2}$, to transform the radial parts of the Schroedinger equations of the two problems into one another [10]. Goded [12] has shown that this is the only substitution of the form $r=\rho^{k}$ which will produce a similar result. The angular parts of the two problems are treated separately, so that the method does not involve the use of a symmetry group.

Classically, the relation of the two problems has been much more tangible. If one attempts to reproduce the analogy just described, he finds that the substitution $r=\rho$ transforms the radial Hamilton-Jacobi equation for the Kepler problem

$$
\partial S / \partial r=\sqrt{2 m^{2} / r+2 m W-\alpha_{\theta}^{2} / r^{2}}
$$

into that of the three dimensional harmonic oscillator

$$
\partial S / \partial \rho=\sqrt{m^{\prime} k \rho^{2}+2 m^{\prime} W^{\prime}-\alpha_{\theta}^{\prime 2} / \rho^{2}} .
$$

However, the separation of the Hamilton-Jacobi equation is commulative, just as it is for the corresponding Schroedinger equation. By this we mean that when $\phi$ is separated, $P_{\phi}$ is seen to be a constant, $\alpha_{\phi}$. When $\alpha_{\phi}$ is placed in the $\theta$ equation another separation constant $\alpha_{\theta}$ is found for $P_{\theta}$, and which finally allows separation of the $r$ equation. Thus the substitution $r=\rho^{2}, P_{r}=f\left(P_{\rho}\right)$ is not easily extended to a canonical transformation involving $P_{\phi}$ and $P_{\theta}$ as well, and it is slightly too naive to say that the transformation $r=\rho^{2}$ transforms the Kepler problem into the harmonic oscillator.

Yet, when $x$ and $y$ lie in the orbital plane, and one defines $r=x+i y$ as a complex variable, the canonical transformation to parabolic coordinates can be expressed in terms of complex variables by just the transformation $r=\rho^{2}$. Thus, in a sense, the square root mapping does relate the two problems.

More correctly, however, one should follow the derivation as given 
in Born [5] or in Wintner [25], in which it is seen that if the motion is uniformized by the introduction of the eccentric anomaly as a time parameter, the transformation to parabolic coordinates and the reduction of the problem to the harmonic oscillator follows quite naturally. Moreover, since the energy appears in the transformation with a square root, the bound and unbound orbits are transformed differently. $R(4)$ applies only to the bound orbits, whereas a Lorentz group $L(4)$ is the symmetry group of the unbound orbits.

Since we are primarily interested in determining a symmetry group for the Kepler problem, it is interesting to note that our precepts have not produced Fock's $R(4)$ group, or the analogous $R(3)$ group for the two dimensional problem, either for parabolic or for polar coordinates, the two systems in which the problem is separable. Rather they have led to respectively an $S U(3)$ and an $S U(2)$ group. Even in the latter case, for which $S U(2)$ and $R(3)$ are locally isomorphic, there is trouble.

The mappings (equations (26)) are valid for functions of the phase space coordinates and momenta. However, if they are taken as operator equations and the operators to be irreducible, then $H$ is a scalar, a nonzero multiple of the unit matrix. The traces of both sides of (26c) cannot vanish, as they must if both $D$ and $D^{\prime}$ are commutators, as they must be on account of the rules given by equations (27). Fortunately in the context of our analysis the eigenvalues of $H$ occur in negative pairs, and thus we would be dealing with reducible representations.

It is already clear from this simple difficulty that while we have introduced a method which will produce an abundance of constants of the motion and symmetry groups for problems of classical mechanics, even such an innocuous, nontranscendental transformation as equations (26) represent, cannot be generalized by use of the correspondence principle for quantum mechanical operators without the exercise of considerable caution.

\section{BIBLIOGRAPHY}

1. A. P. Alliluev, On the relations between accidental degeneracy and hidden symmetry of a system, Soviet Physics JETP 6 (1958), 156-159. (Journal Experimental and Theoretical Physics (USSR) 33 (1957), 200-203.)

2. V. Bargmann, Zur Theorie des Wasserstoffatom, Zeitschrift für Physik 99 (1936), $576-582$.

3. L. C. Biedenharn, Remarks on the relativistic Kepler problem, Physical Review 126 (1962), 845-651.

4. L. C. Biedenharn and N. V. V. J. Swamy, Remarks on the relativistic Kepler problem. II Approximate Dirac-Coulomb Hamiltonian prossessing two vector invariants, Physical Review 133 (1964), B 1353-B 1360.

5. M. Born, The Mechanics of the Atom, Frederick Ungar Pub. Co., New York, 1960.

6. Yu. N. Demkov, The definition of the symmetry group of a quantum system. The 
anisotropic oscillator, Soviet Physics JETP 17 (1963), 1349-1351; (JETP (USSR) 44 (1963), 2007-2010).

7. V. A. Dulock and H. V. McIntosh, Degeneracy of cyclotron motion, J. Math. Physics 7 (1966).

8. V. A. Dulock and H. V. McIntosh, On the degeneracy of the two dimensional harmonic oscillator, Amer. J. Physics 33 (1965), 109-118.

9. V. Fock, Zur Theorie des Wasserstoffatoms, Zeitschrift fur Physick 98 (1935), 145-154.

10. W. Furry, Private communication.

11. C. S. Gardner, Adiabatic invariants of periodic classical systems, Physical Review 115 (1959), 791-794.

12. F. Goded, Transformations of the Schroedinger equation with discrete energy spectrum, Il Nuovo Cimento 26 (1962), 1346-1353.

13. E. L. Hill, Seminar on the Theory of Quantum Mechanics, (unpublished), University of Minnesota, 1954.

14. L. Hulthen, Uber die quantenmechanische Herleitung der Balmerterme, Zeitschrift für Physik, 86 (1933), 21-23.

15. J. M. Jauch and E. L. Hill, On the problem of degeneracy in quantum mechanics, Physical Review 57 (1940), 641-645.

16. O. Laporte and G. Y. Rainich, Stereographic parameters and pseudo-minimal hypersurfaces, Trans. Amer. Math. Soc. 39 (1936), 154-182.

17. W. Lenz, Uber das Bewgungsvergriff und die Quantenzuständ der gestörten Keplerbewegung, Zeitschrift für Physik 24 (1924), 197-207.

18. R. Loudon, One-dimensional hydrogen atom, Amer. J. Physics 27 (1959), 649-655.

19. W. Magnus and F. Oberthettinger, Formulas and Theorems for the Special Functions of Mathematical Physics, Chelsea Pub. Co., New York, 1949.

20. M. A. Melvin and N. V. V. J. Swamy, Evaluation of certain physically interesting integrals and hypergeometric sums, Journal of Mathematics and Physics 36 (1957), 157-163.

21. W. Pauli, Uber das Wasserstoffspektrum von Standpunkt der neuen Quantenmechanike, Zeitschrift für Physik 36 (1926), 336-363.

22. P. J. Redmond, Generalization of the Runge-Lenz vector in the presence of an electric field, Physical Review 133 (1964), B 1352-B 1353.

23. A. W. Saenz, On Integrals of the Motion of the Runge Type in Classical and Quantum Mchanics, Ph. D. Thesis, University of Michigan, 1949.

24. E. T. Whittaker, Analytical Dynamics, Cambridge University Press, 1961.

25. A. Wintner, The Analytical Foundations of Celestial Mechanics, Princeton University Press, 1947.

Received September 17, 1965. Based on part of a Dissertation submitted to the Graduate School of the University of Florida in partial fulfillment of the requirements for the Ph. D. degree. Supported in part by Air Force Grant AFOSR-471-64.

Department OF Physics

UNIVERSITY OF FLORIDA

GAINESVILLE, FLORIDA 



\section{PACIFIC JOURNAL OF MATHEMATICS}

\section{EDITORS}

\author{
H. SAMELSON \\ Stanford University \\ Stanford, California \\ J. P. JANS \\ University of Washington \\ Seattle, Washington 98105
}

\author{
J. DUGUNDJI \\ University of Southern California \\ Los Angeles, California 90007 \\ RICHARD ARENS \\ University of California \\ Los Angeles, California 90024
}

\section{ASSOCIATE EDITORS}
E. F. BECKENBACH
B. H. NeumanN
F. WOLF
K. YOSIDA

\section{SUPPORTING INSTITUTIONS}

\author{
UNIVERSITY OF BRITISH COLUMBIA \\ CALIFORNIA INSTITUTE OF TECHNOLOGY \\ UNIVERSITY OF CALIFORNIA \\ MONTANA STATE UNIVERSITY \\ UNIVERSITY OF NEVADA \\ NEW MEXICO STATE UNIVERSITY \\ OREGON STATE UNIVERSITY \\ UNIVERSITY OF OREGON \\ OSAKA UNIVERSITY \\ UNIVERSITY OF SOUTHERN CALIFORNIA
}

STANFORD UNIVERSITY

UNIVERSITY OF TOKYO

UNIVERSITY OF UTAH

WASHINGTON STATE UNIVERSITY

UNIVERSITY OF WASHINGTON

AMERICAN MATHEMATICAL SOCIETY CHEVRON RESEARCH CORPORATION TRW SYSTEMS

NAVAL ORDNANCE TEST STATION 


\section{Pacific Journal of Mathematics}

\section{Vol. 19, No. 1 \\ May, 1966}

A. R. Brodsky, The existence of wave operators for nonlinear equations... 1

Gulbank D. Chakerian, Sets of constant width................... 13

Robert Ray Colby, On indecomposable modules over rings with minimum condition....................................... 23

James Robert Dorroh, Contraction semi-groups in a function space ....... 35

Victor A. Dulock and Harold V. McIntosh, On the degeneracy of the Kepler

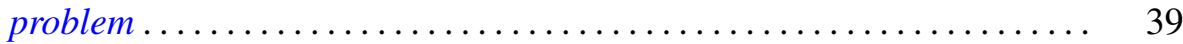

James Arthur Dyer, The inversion of a class of linear operators ......... 57

N. S. Gopalakrishnan and Ramaiyengar Sridharan, Homological dimension of Ore-extensions ................................. 67

Daniel E. Gorenstein, On a theorem of Philip Hall ................. 77

Stanley P. Gudder, Uniqueness and existence properties of bounded observables..................................... 81

Ronald Joseph Miech, An asymptotic property of the Euler function ....... 95

Peter Alexander Rejto, On the essential spectrum of the hydrogen energy and related operators ............................... 109

Duane Sather, Maximum and monotonicity properties of initial boundary

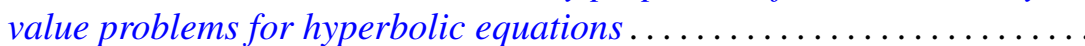

Peggy Strait, Sample function regularity for Gaussian processes with the parameter in a Hilbert space ........................... 159

Donald Reginald Traylor, Metrizability in normal Moore spaces ........... 175

Uppuluri V. Ramamohana Rao, On a stronger version of Wallis' formula ...............................

Adil Mohamed Yaqub, Some classes of ring-logics....... 